\title{
Schistosomiasis mansoni and soil-transmitted helminthiasis in Bushulo village, southern Ethiopia
}

\author{
Ashenafi Terefe ${ }^{1}$, Techalew Shimelis ${ }^{2}$, Mulugeta Mengistu ${ }^{2}$, Asrat Hailu $^{3}$, Berhanu Erko ${ }^{4}$
}

\begin{abstract}
Background: Schistosomiasis mansoni and soil-transmitted helminthiasis (STHs) are considerable medical and public health problems in Ethiopia. However, information is limited on the epidemiology of these infections in different localities even though it is needed to plan effective prevention and control measures.

Objective: This study was designed to determine the prevalence of Schistosoma mansoni and soil-transmitted helminth infections in school children and residents in Bushulo village near Lake Awassa (Hawassa), southern Ethiopia.

Methods: Cross-sectional epidemiological and parasitological studies were conducted on schistosomiasis mansoni and STHs in Bushulo village in May and June 2007. A total of 419 participants (353 school children and 66 other residents) were included in the study. The principal investigator interviewed the study subjects about demographic status using structured questionnaires. Moreover, experienced nurse took history and conducted physical examination to assess symptoms and signs related to chronic $S$. mansoni infection. A single stool sample was collected from each participant and processed using the Kato-Katz technique. Experienced laboratory technician read all slides at Bushulo Health Center.

Results: The overall infection rates of schistosomiasis mansoni, trichuriasis, ascariasis and hookworm infection were $73.7 \%, 41.5 \%, 37.2 \%$ and $28.4 \%$, respectively. Other parasitic infections observed were caused by Hymenolepis nana (1.7\%), Taenia species (1.4\%), and Enterobius vermicularis $(1.4 \%)$. Children in the age range $10-14$ years and those attending at St. Paul's School had higher rates of T. trichiura and S. mansoni, respectively. Intensity of infection was higher for A. lumbricoides in the age range 5-9 years. The overall prevalence of any STHs was $67.3 \%$. The rates of single, dual, triple and quadruple infections were $29.6 \%, 32 \%, 20.3 \%$ and $7.4 \%$, respectively.

Conclusion: The high prevalence and intensity of schistosomiasis mansoni and soil-transmitted helminthiasis makes periodic deworming programme urgent to reduce morbidity and transmission of helminthiasis in the area. Provisions of sanitary facilities and clean water supply as well as health education are also critically needed to sustain the impact of chemotherapy. [Ethiop. J. Health Dev. 2011;25(1):46-50]
\end{abstract}

\section{Introduction}

Schistosomiasis and soil-transmitted helminthiasis are widely distributed and are among the major medical and public health problems in many parts of the world. According to the World Health Organization (WHO), about 1.45 billion people globally are infected with Ascaris lumbricoides, 1.3 billion with hookworm and 1.05 billion with Trichuris trichiura (1). Schistosomiasis is also common in many parts of the world and about 200 million people are infected globally (2).

Intestinal helminthiasis is intimately related to poverty and most infections are entrenched particularly in tropical regions where the biophysical environment and cultural practices favor transmission (3). For instance, of the global burden of schistosomiasis, an estimated $85 \%$ is found in sub-Saharan Africa (2). Infection with helminths is responsible for many deaths and morbidities. Among tropical parasitic diseases, the yearly estimated deaths of 200,000 are making schistosomiasis second only to malaria as a cause of mortality (2). Apart from morbidity and mortality, intestinal helminthiasis is associated with poor physical growth and development, as well as with retarded intellectual and cognitive development in children $(4,5)$.

In Ethiopia, numerous epidemiological surveys of intestinal helminths were conducted and varying infection rates were reported from different regions and communities (6-9). However, most of past studies overlooked estimating intensity of infections, which would have been crucial for epidemiological and clinical evaluations. Unsafe and inadequate provision of water, unhygienic living conditions and unsanitary waste management allow intestinal parasites and other communicable diseases to flourish in various localities (10). S. mansoni has been recorded from all regions and is rapidly spreading with water resource development and population movements (11). S. mansoni is transmitted by Biomphalaria pfeifferi in most endemic foci in Ethiopia and by $B$. sudanica in only three foci (12).

1School of Medical Laboratory Technology, Jimma University, E-mail ashenafidejene@gmail.com, P.O. Box 378, Jimma, Ethiopia;

2Department of Medical Laboratory Science, Hawassa University, P.O. Box 1560, Hawassa, Ethiopia;

3Department of Microbiology, Immunology and Parasitology, Faculty of Medicine, Addis Ababa, University, Addis

Ababa, Ethiopia;

4Aklilu Lemma Institute of Pathobilogy, Addis Ababa University, P.O. Box 1176, Addis Ababa, Ethiopia 
Schistosomiasis mansoni and soil-transmitted helminthiasis are among the major health problems around Lake Awassa (Hawassa) in southern Ethiopia. However, little is known regarding the epidemiology of these infections around Lake Awassa, where majority of children are involved in fishing and swimming activities. This survey was, therefore, conducted to determine the prevalence and intensity of $S$. mansoni and soiltransmitted helminthes infections in Bushulo village near Awassa Lake, to provide baseline information for future prevention and control programs.

\section{Methods}

Cross-sectional epidemiological and parasitological studies were conducted in Bushulo village in May and June 2007. Bushulo is a small rural town, found in Southern Nations and Nationalities Peoples Region and located along the shore of the Lake Awassa. It is located at 1,700 meters above sea level with temperatures ranging from $11.5{ }^{\circ} \mathrm{C} \quad-33{ }^{\circ} \mathrm{C} \quad$ (13). Social service institutions in the area include, one major health center and five schools (1 kindergarten and 4 junior secondary schools). The health center had beds and provided diagnostic and treatment services for its five catchment kebeles (administrative units) of Tulo, Alamura, Finchuwa, Worancha and St. Paulo's. Few standing pipes and dug wells were supplying water for residents in the village although they are inadequate to meet their demands. Thus, inhabitants largely relied on water from Lake Awassa for both domestic and laundering purposes. As to what proportion of inhabitants had and used latrines properly was not known. During this survey, however, we observed areas nearby bushes and the lakeside polluted with human faeces.

The study population consisted of school children attending grades 2-7 in three junior secondary schools (Tulo, St. Paulo's, and Finchuwa) and residents of Worancha Kebele. Selection of these schools and the Worancha Kebele was based on the presence of water contact points along the shore of the lake. The study subjects were those who had lived in the area for 3 or more years and had not taken treatment for any intestinal ailment during the month prior to the survey.

Sample size was estimated only for school children on the basis of S. mansoni prevalence of 33\% (14), precision of $5 \%$ and $95 \%$ confidence interval. Rosters at the 3 schools were used as a sampling frame and a systematic sampling technique was employed to include school children (Tulo, St. Paulo's, and Finchuwa) as study subjects. Studying school population probably results in over-reporting of infections levels because of the higher rates in younger age groups. Hence, all members above 5 years of age from 20 randomly selected households in Worancha Kebele were also included for comparative purposes. It has to be said that, constraint of resources impeded including a justified number of residents. In total, 419 individuals (353 children and 66 residents) participated in this study.
The principal investigator interviewed the study subjects about their demographic status using structured questionnaires. Moreover, an experienced nurse took history and carried out physical examination to assess symptoms and signs related to chronic $S$. mansoni infection. Only symptoms present within 2 weeks of examination and signs present at the date of investigation were included in the analyses. A single stool sample was collected from each participant, and two slides per sample were prepared using the Kato-Katz technique at respective schools and the Kebele (3). On the same day of stool collection, slides were transferred to Bushulo Health Center and read once by an experienced laboratory technician. Averages of egg count on pair of slides per sample were taken and conversions into eggs per gram (epg) of stool were done by a factor of 24 . Intensity of infection for each parasite was estimated using epg and categorized as light, moderate and heavy infection according to the WHO criteria specified as follows: S. mansoni [Light (1-99 epg), moderate (100399 epg), heavy ( $\geq 400 \mathrm{epg})]$; T. trichiura [Light (1-999 epg), moderate (1000-9999 epg), heavy ( $\geq 10,000$ epg)]; A. lumbricoides [Light (1-4999 epg), Moderate (5,00049,999 epg), Heavy ( $\geq 50,000$ epg)] (1).

The study was approved by the Ethical clearance Committee of the Faculty of Medicine, Addis Ababa University. Participation was fully voluntary and study subjects or their parents gave informed oral consent. Participants found to be positive for any intestinal parasite were treated with anti-parasitic drugs as recommended by WHO (1).

The data were analyzed using SPSS Version-13 software and results were summarized using means and percentages. Pearson Chi-square test and One-Way Anova were used to evaluate differences in proportions and means, respectively. A p-value $<0.05$ was considered to be significant.

\section{Results}

A total of 419 individuals participated in this study 353 of whom were children attending the 3 study schools (Tulo, St. Paulo's and Finchuwa) and 66 were residents of Worancha Kebele. Most of the participants $(92.4 \%)$ were under 20 years of age (the mean being 13.2 years) and the male to female ratio was $1: 0.8$.

Microscopic examination of stool samples showed that $323(91.5 \%)$ of the school children and $51(77.3 \%)$ of the residents were positive for at least 1 parasite. Seven helminth parasite species were identified and the most prevalent were $S$. mansoni (73.7\%), followed by $T$. trichiura (41.5\%), A. lumbricoides (37.2\%) and hookworm species $(28.4 \%)$ (Table 1$)$. The prevalence of any STHs was $67.3 \%$. Rare parasites observed were Hymenolepis nana (1.7\%), Taenia species (1.4\%), and Enterobius vermicularis (1.4\%), although Teania infections were characteristically underreported in stool tests.

Ethiop J Health Dev 2011;25(1) 
S. mansoni was significantly associated with the study sites $(\mathrm{p}=0.002)$, and the highest infection rates manifested in children attending St. Paul's School $(84.3 \%)$. Though differences were non-significant, rates of infection were higher among children in the age group $10-19$ years $(77.6 \%)$ and in males $(76.6 \%)$. Moreover, $T$. trichiura was significantly associated with age $(\mathrm{p}=0.02)$, and the highest infection rates were observed in children 10 -14 years old $(46.5 \%)$ (Table 1$)$.
The intensity of infection was determined for $A$. lumbricoides, T. trichiura and S. mansoni. Although high intensities of $S$. mansoni infection were observed among the participants, associations of age, gender and study site with an arithmetic mean epg were not significant. However, intensities of $A$. lumbricoides infection were significantly influenced by age $(\mathrm{p}=0.03)$, with the highest mean epg observed in children 5-9 years old $(13,236$ epg) (Table 2).

Table 1: Prevalence of schistosomiasis mansoni and soil-transmitted helminthiasis by age, gender and study sites at Bushulo village, southern Ethiopia, 2007

\begin{tabular}{|c|c|c|c|c|c|}
\hline Characteristics & $\begin{array}{l}\text { Total No. } \\
\text { examined }\end{array}$ & $\begin{array}{l}\text { S. mansoni no. } \\
(\%)\end{array}$ & $\begin{array}{l}\text { T. trichiura } \\
\text { No. (\%) }\end{array}$ & $\begin{array}{l}\text { A. lumbricoides } \\
\text { No. (\%) }\end{array}$ & $\begin{array}{l}\text { Hookworm } \\
\text { No. (\%) }\end{array}$ \\
\hline \multicolumn{6}{|l|}{ Age (years) } \\
\hline $5-9$ & 115 & $78(67.8)$ & $50(43.5)$ & $43(37.4)$ & $35(30.4)$ \\
\hline $10-14$ & 200 & $156(78.0)$ & $93(46.5)$ & $84(42.0)$ & $53(26.5)$ \\
\hline $15-19$ & 72 & $55(76.4)$ & $19(26.4)$ & $20(27.8)$ & $22(30.6)$ \\
\hline $20+$ & 32 & $20(62.5)$ & $12(37.5)$ & $9(28.1)$ & $9(28.1)$ \\
\hline Total & 419 & $309(73.7)$ & $174(41.5)$ & $156(37.2)$ & $119(28.4)$ \\
\hline$p$-value & & 0.10 & 0.02 & 0.12 & 0.86 \\
\hline \multicolumn{6}{|l|}{ Gender } \\
\hline Male & 218 & $167(76.6)$ & $95(43.6)$ & $89(40.8)$ & $70(32.1)$ \\
\hline Female & 201 & $142(70.6)$ & 79 (39.3) & $67(33.3)$ & $49(24.4)$ \\
\hline$p$-value & & 0.20 & 0.42 & 0.33 & 0.10 \\
\hline \multicolumn{6}{|l|}{ Study sites } \\
\hline Tulo & 148 & $108(73.0)$ & $70(47.3)$ & $62(41.9)$ & $51(34.5)$ \\
\hline St. Paulo's & 121 & $102(84.3)$ & $40(33.1)$ & $40(33.1)$ & $32(26.4)$ \\
\hline Finchwua & 84 & $60(71.4)$ & $37(44.0)$ & $34(40.5)$ & $18(21.4)$ \\
\hline Worancha & 66 & $39(59.1)$ & 27 (40.9) & $20(30.3)$ & $18(27.3)$ \\
\hline p-value & & 0.002 & 0.12 & 0.26 & 0.17 \\
\hline
\end{tabular}

Table 2: Arithmetic mean eggs per gram (epg) of stool for S. mansoni, A. Iumbricoides, and T. trichiura infections by age and gender in Bushulo village, southern Ethiopia, 2007.

\begin{tabular}{clcc}
\hline Characteristics & $\begin{array}{c}\text { S. mansoni } \\
\text { Mean epg }\end{array}$ & $\begin{array}{c}\text { T. trichiura } \\
\text { Mean epg }\end{array}$ & $\begin{array}{c}\text { A. Iumbricoides } \\
\text { Mean epg }\end{array}$ \\
\hline Age (years) & & & \\
$5-9$ & 434.1 & 74.9 & 13236.0 \\
$10-14$ & 534.8 & 126.2 & 3022.9 \\
$15-19$ & 597.3 & 57.0 & 536.7 \\
$20+$ & 246.0 & 229.9 & 3995.8 \\
\hline p-value & $\mathbf{0 . 1 7}$ & $\mathbf{0 . 3 1}$ & $\mathbf{0 . 0 3}$ \\
\hline Gender & & & \\
Male & 643.3 & 90.7 & 8021.1 \\
Female & 336.4 & 127.9 & 2420.2 \\
\hline p-value & $\mathbf{0 . 1 9}$ & $\mathbf{0 . 2 0}$ & $\mathbf{0 . 2 2}$ \\
\hline
\end{tabular}

As presented in Table 3, the majority of the study subjects harboring A. lumbricoides or T. trichiura had light infections. Heavy infections were common among subjects with $S$. mansoni infections (42.5\%); no case of heavy infection with $T$. trichiura was observed.

Participants were infected with 1-4 parasites. Multiple infections (polyparasitism) were seen in 250 (59.7\%) of all 419 examined individuals and in $66.8 \%$ of those with any infection (374 individuals). Most parasites occurred as double infections (32\%) (Table 4). S. mansoni most frequently combined with $T$. trichiura and $A$. lumbricoides occurred in 85 individuals (20.3\%) as a triple infection. Moreover, these three parasites combined with hookworm species in 31 individuals (7.4\%) as quadruple infection. The prevalence of mixed infection was not affected by age, gender or study sites.

Table 5 summarizes the prevalence of signs and symptoms among the participants stratified by $S$. mansoni infections. The most frequently reported symptoms in the study subjects were recurrent abdominal 
discomfort (46.7\%) and diarrhea (29.1\%). A history of diarrhea was significantly higher among participants with S. mansoni compared to those without this infection $(\mathrm{p}=$ $0.01)$.

Table 3: Prevalence and intensity of S. mansoni, A. lumbricoides, and $T$. trichiura infections among persons with respective parasite infected in Bushulo village, southern Ethiopia, 2007

\begin{tabular}{lrc}
\hline Parasite & Frequency & Percent \\
\hline S. mansoni & & \\
$\quad$ Light (1-99 epg) & 76 & 24.6 \\
$\quad$ Moderate (100-399 epg) & 103 & 33.3 \\
$\quad$ Heavy ( $\geq 400 \mathrm{epg})$ & 130 & 42.1 \\
T. trichiura & & \\
$\quad$ Light (1-999 epg) & 163 & 93.7 \\
$\quad$ Moderate (1000-9999 epg) & 11 & 6.3 \\
$\quad$ Heavy ( $\geq 10,000 \mathrm{epg})$ & 0 & 0 \\
A. lumbricoides & & \\
Light (1-4999 epg) & 110 & 70.5 \\
Moderate (5,000-49,999 epg) & 38 & 24.3 \\
$\quad$ Heavy ( $\geq 50,000 \mathrm{epg})$ & 8 & 5.1 \\
\hline
\end{tabular}

Table 4: Prevalence of single and multiple infections by sex in Bushulo village, southern Ethiopia, 2007.

\begin{tabular}{lccc}
\hline \multirow{2}{*}{ Infection status } & \multicolumn{3}{c}{ Number $(\%)$ of individuals } \\
\cline { 2 - 4 } & Males & Females & Total \\
\hline No parasites & $19(8.7)$ & $26(12.9)$ & $45(10.7)$ \\
Single infection & $61(28.0)$ & $63(31.3)$ & $124(29.6)$ \\
Double infections & $66(30.3)$ & $68(33.8)$ & $134(32.0)$ \\
Triple infections & $50(22.9)$ & $35(17.4)$ & $85(20.3)$ \\
Quadruple & $22(10.1)$ & $9(4.5)$ & $31(7.4)$ \\
infections & & & \\
\hline
\end{tabular}
infections

Table 5: Prevalence of signs and symptoms stratified by S. mansoni infection status in Bushulo village, southern Ethiopia, 2007

\begin{tabular}{lcccc}
\hline $\begin{array}{l}\text { Signs and } \\
\text { symptoms }\end{array}$ & $\begin{array}{l}\text { Positive for S. } \\
\text { mansoni } \\
(\mathbf{N = 3 0 9 )} \text { No. (\%) }\end{array}$ & $\begin{array}{l}\text { Negative for S. mansoni } \\
(\mathbf{N}=110)\end{array}$ & $\begin{array}{l}\text { Total }(\%) \\
\text { No. (\%) }\end{array}$ & p-value \\
\hline Enlarged liver & $10(3.2)$ & $2(1.8)$ & $12(2.9)$ & 0.70 \\
Enlarged spleen & $15(4.9)$ & $3(2.7)$ & $18(4.3)$ & 0.52 \\
Blood in stool & $22(7.1)$ & $4(3.6)$ & $26(6.2)$ & 0.19 \\
Abdominal pain & $151(48.9)$ & $45(40.9)$ & $196(46.7)$ & 0.15 \\
Diarrhea & $80(25.9)$ & $42(38.2)$ & $122(29.1)$ & 0.01 \\
\hline
\end{tabular}

\section{Discussion}

We determined the prevalence and intensity of infection with $S$. mansoni and soil-transmitted helminths among school children and residents of Bushulo village along the shore of Lake Awassa. The overall prevalence of helminth infection in this study was $89.3 \%$ and infection rates were very high in children in all 3 schools and in residents of Worancha Kebele. The present study showed infection rates comparable with those previously reported from other communities in the Rift Valley in southern Ethiopia (14-16).

In Ethiopia, altitude between 1,200 to 2,000 meters is optimal for S. mansoni transmission and the majority of endemic areas are located in this altitudinal range (11). High infection rates of $S$. mansoni above $50 \%$ were reported in the hyperendemic areas of northwestern (Gorgora, Zerima, and Dek) (6), northeastern (Kemise and Bati towns) (7) and northern Ethiopia (Adawa) (8). However, previous studies on populations living in the same high-risk altitudinal zone in the southern part of the country reported moderate prevalence rates of $S$. mansoni infection $(14,15,17,18)$. The high prevalence and intensity of $S$. mansoni and other parasites in the present study may be attributable to unhygienic conditions, inadequate provision of water and unsanitary waste management that, in turn, forcing residents to resort to the usage of the lake water. The magnitude of the problem emphasizes the need to take immediate intervention measures. Combined mass chemotherapy and focal snail control using primary health care systems reduced $S$. mansoni infection significantly in western Ethiopia (19). Similar interventions may have an effect on the prevalence and intensity of infections in the study area.

The high prevalence and mean intensity of $S$. mansoni infection, regardless of the age and gender of participants, highlights the negative impact it might have to promote further transmission as well as getting enormous clinical significance in various segments of the population. Likewise, high intensity of infection was reported from northern Ethiopia (8). In contrast to the present study, however, lower intensities were reported by others $(6,17)$. $S$. mansoni infection was significantly influenced by study sites, with the highest rate observed in children attending St. Paul's School. This may be due to the nearness of the children's dwelling close to the lake, which enhances exposure to infected water although this was not systematically studied. 
The prevalence of ascariasis and hookworm infection was also in accordance with the rates reported for the 1,000 to $2,000 \mathrm{~m}$ zone in Ethiopia (10). Similar prevalence of $A$. lumbricoides and $T$. trichiura is possibly due to their shared ecology and life cycle. The higher intensity of ascariasis among children 5-9 years old may be because they indiscriminately play on faecally contaminated grounds and have a common habit of placing soiled fingers in the mouth.

Multiple infections are common in Ethiopia $(15,16)$. In this study, 1 to 4 species of parasites per individual were observed and of those with any infection most individuals harbored more than 1 parasite. This may be because of the higher prevalence of each parasites and/or their similar mode of transmission which favors multiple infections.

In conclusion, the high prevalence and intensity of schistosomiasis mansoni and soil-transmitted helminthiasis observed in the study area call for a periodic deworming programme to reduce transmission, worm burden and morbidity. Deworming should be supplemented with improved sanitation and access to clean water, appropriate health education and environmental measures to have a lasting impact on transmission.

\section{Acknowledgments}

The authors would like to thank the technical staff of Aklilu Lemma Institute of Pathbiology for their assistance in data collection. We also thank the community of Bushulo Village, who volunteered to participate in this study and finally, the Department of Microbiology, Immunology and Parasitology, Addis Ababa University for financial and logistic support for the study.

\section{References}

1. World Health Organization (WHO). Prevention of Schistosomiasis and Soil Transmitted Helminthiasis: Report of WHO Expert Committee. WHO Technical Report Series 912. Geneva; 2002.

2. World Health Organization Weekly Epidemiologic Record 2006, 81, 145-164 http://www.who.int/wer/ 2006/wer8116.pdf (accessed 23/10/2009).

3. Cheesbrough M. District Laboratory Practice in Tropical Countries, 2nd ed., Part 1, Cambridge University Press, UK, 2000.

4. Nokes C, Bundy DAP. Does helminth infection affect mental processing and educational achievement? Parasitol Today 1994; 10:14-8.

5. Nokes C, Cooper ES, Robinson BA, Budy DAP. Geohelminth infection and academic assessment in Jamaican children. Trans Roy Soc Trop Med Hyg $1991 ; 85: 272-3$.

6. Tiruneh M, Fantahun M, Kassu A, Tiruneh G, Van Lieshout L. Schistosomiasis mansoni in school attenders and non-attenders in Northwest Ethiopia. Ethiop J Health Dev 2001; 15:117-23.
7. Birrie H, Abebe F, Gundersen SG, Medhin G, Berhe $\mathrm{N}$, Gemetchu T. Epidemiology of schistosomiasis mansoni in three endemic communities in north-east Ethiopia: baseline characteristics before endod based intervention. Ethiop Med J 1998; 36:101-11.

8. Birrie H, Woldemichael T, Redda A, Chane T. The status of Schistosoma mansoni and snail hosts in Tigray and northern Wello regions, northern Ethiopia. Ethiop Med J 1994; 32:245-54.

9. Mengistu A, Gebre-Selassie S, Kassa T. Prevalence of intestinal parasitic infections among urban dwellers in southwest Ethiopia. Ethiop $J$ Health Dev 2007;21(1):12-17.

10. Habtamu B, Kloos $\mathrm{H}$ : Intestinal parasitism. In: Berhane Y, Hailemariam D, Kloos H. eds. The Epidemiology and Ecology of Health and Diseases in Ethiopia. Addis Ababa: Shama Books; 2006: 51938.

11. Erko B, Abebe F, Berhe N, et al. Control of Schistosoma mansoni by the soapberry Endod (Phytolacca dodecandera) in Wollo, Northeastern Ethiopia post intervention prevalence. East Afr Med $J$ 2002; 79:198-201.

12. Ali A, Erko B, Woldemichael $\mathrm{T}$, Kloos $\mathrm{H}$ : Schistosomiasis. In: Berhane Y, Hailemariam D, Kloos H. (eds). The Epidemiology and Ecology of Health and Diseases in Ethiopia. 1st edition. Addis Ababa: Shama books; 2006: 660-73.

13. Hawassa City Administration. Socioeconomic profile 1996-1998 E.C. Hawassa, 1999 E.C.

14. Merid Y, Hegazy M, Mekete G, Teklemariam S. Intestinal helminthic infection among children at Lake Awassa Area, South Ethiopia. Ethiop J Health Dev 2001; 15:31-38.

15. Legesse M, Erko B. Prevalence of intestinal parasites among school children in a rural area close to Lake Langano in the southeast Ethiopia. Ethiop $J$ Health Dev 2004; 18: 116-20.

16. Roma B, Worku S. Magnitude of schistosoma mansoni and intestinal helminthic infections among school children in Wondo Genet Zuria, Southern Ethiopia. Ethiop J Health Dev 1997; 11:125-29.

17. Erko B, Medhin G, Berhe N, Abebe F, GebreMichael T, Gundersen SG. Epidemiological studies on intestinal schistosomiasis in Wondo Genet, southern Ethiopia. Ethiop Med J 2002; 40:29-39.

18. Erko B, Medhin G. Human helminthiasis in Wondo Genet, southern Ethiopia, with emphasis on geohelminthiasis. Ethiop Med J 2003; 41(4):333-44.

19. Gundersen SG, Birrie H, Torvik HP et al. Control of Schistosoma mansoni in the Blue Nile Vally of western Ethiopia by mass chemotherapy and focal snail control: primary health care experience. Trans Roy Soc Trop Med Hyg 1990; 84:819-25. 